\title{
COLLABORATIVE HOME MEDIA COMMUNITY WITH SEMANTIC SUPPORT
}

\author{
Hongguang Zhang, Marc Girod Genet, Djamal Zeghlache, Tien Anh Le, Hang Nguyen, Noel Crespi \\ Institut Telecom, Telecom SudParis \\ Email: \{hongguang.zhang, marc.girod_genet, djamal.zeghlache, tien_anh.le, hang.nguyen, \\ noel.crespi\}@it-sudparis.eu
}

\begin{abstract}
The great success of social technologies such as media sharing, blogs and wikis, is transforming the Internet into a collaborative community. This paper presents our research towards the exploitation of P2P networks, semantic metadata and social tagging for home media sharing, with a vision of P2P-based Collaborative Home Media Community (CHMC). The goal of the proposed CHMC is to enable sharing, searching and tagging of multimedia contents based on semantic metadata within home networks as well as between homes connected by broadband networks. We will firstly present a hierarchical P2P network architecture where the super-peer model is applied. The super peers act as a gateway bridging the inner home network with the external Internet. We further propose the Resource Description Framework (RDF) triple-based metadata indexing, retrieving and tagging algorithms on structured P2P networks. Finally, a prototype is developed for the purposes of system validation and performance evaluation.
\end{abstract}

Keywords - Media Sharing, Semantic Metadata, Social Tagging, P2P Overlay

\section{INTRODUCTION}

With the development of digital technologies, the home network is paving the way for significant enhancements to the user's entertainment experience [1]. As a result, the demand of digital media is growing rapidly in home. Meanwhile, the proliferation of mobile devices and wireless networks makes multimedia be ubiquitous and all-pervasive beyond the scope of home. The ubiquitous multimedia results in large personal and public multimedia database in which it has become difficult to retrieve specific contents and browse large collections. Semantic metadata is a solution to the overwhelming resources. However, the lack of semantic metadata is becoming a barrier for the in-depth study and wide application. Recently, the success of social networking has shown that user experiences are enriched by

\footnotetext{
This work was supported by the ITEA-2 CAM4Home project.
}

sharing, aggregating, and tagging collaboratively. Under this trend, folksonomy also known as social tagging or collaborative annotation draws more and more attention as a promising source of semantic metadata.

Home media related topics have been hot in both industries and academia for many years, which results in relevant standardization activities. Universal Plug and Play (UPnP) [2] allows devices to connect seamlessly and to simplify the implementation of networks in the home for data sharing, communication and entertainment. The Digital Living Network Alliance (DLNA) [3] has been established to provide interoperability among various consumer electronics, PC and mobile devices for media sharing across the digital home environment. However, most of the efforts were made for media sharing among interoperable consumer electronic devices within the home network [4-7]. During the past decade, Peer-to-Peer (P2P) networks have evolved to become one of the most popular means of data retrieval, which is popularized by the large scale file-sharing systems. Based on a scalable distributed architecture, P2P networks are born with the instinct of collaboration between peers, which makes it easy for everyone to create, reuse and share digital media without costly servers and complicated system management. The use of P2P technology has been extended into home media environment. The "Share it!" project $[8,9]$ developed an end-to-end system of home media sharing between homes connected by the broadband Internet in a P2P network of set-top boxes with mass storage. The P2PFUSION project [10] develops a new P2P-based software system that supports audiovisual creative activities and makes it easy for anyone to create, reuse and share audio and video contents. P2P-Next [11] is a project for P2P-based digital media distribution that is intended to produce a new video delivery platform running on a wide range of consumer devices. However, considering the overwhelming multimedia resources, we argue that it is more crucial to support the advanced ability for content discovery compared with P2P-based content delivery. In addition, the deployment of P2P networks faces new challenges in the home network environment where there could be heterogeneous devices ranging from $\mathrm{PC}$ to mobile phones.

In this paper, we propose the concept of Collaborative Home Media Community (CHMC) with the vision of sharing, searching and tagging multimedia contents on the 
level of semantic metadata in home media environment. Making use of structured P2P as the network infrastructure for multimedia sharing, the proposed system is characterized by scalability, collaboration, aggregation, and social tagging. The multimedia semantic metadata plays a vital role in CHMC. We adopt CAM4Home (Collaborative Aggregated Multimedia for Digital Home) framework [12] as the metadata model. Multimedia contents to be shared in CHMC are encapsulated as CAM4Home metadata entities. On the base of CAM4Home model, semantic-coherent entities can be further aggregated into a bundle. CHMC provides the support of social tagging to enrich metadata description collaboratively as well. The proposed CHMC system follows a hierarchical P2P architecture where the super-peer model is used. Furthermore, we propose the algorithms indexing the CAM4Home entities semantically over structured $\mathrm{P} 2 \mathrm{P}$ networks for efficiently querying and searching. To be noted, this paper aims to present a new system for content discovery with the use of semantic metadata, where the specific way for content delivery is out of the discussion. CHMC can be used as an independent component complementary to existing content delivery systems for the retrieval of media contents.

The rest of paper is organized as follows. Section 2 overviews the CAM4Home metadata model. Section 3 presents the P2P-based CHMC service and network architecture. Section 4 proposes the Resource Description Framework (RDF) triple-based indexing, tagging and searching algorithm. The implementation of prototype system is briefly introduced in Section 5. Finally, Section 6 summarizes the paper and overviews the future work.

\section{CAM4HOME METADATA}

The CAM4Home is an ITEA2 project implementing the concept of Collaborative Aggregated Multimedia (CAM). This concept refers to aggregation and composition of individual multimedia content and content-based service entities into a bundle that can be delivered as a semantically coherent composition of content and related services [12]. CAM4Home focuses on specifying a metadata framework enabling a novel way of content and service provisioning by bundling different types of content and service into bundles on the level of metadata. The CAM4Home metadata model supports the representation of a wide variety of multimedia content and service in CAM Element as well as its descriptive metadata. CAM Object is the integrated representation of CAM Element and CAM Element Metadata on the association rule "isMetadataOf'. The metadata model also presents the mechanisms aggregating CAM Objects. CAM Bundles are the aggregation of two or more CAM Objects and a description of that aggregation. Fig. 1 illustrates the CAM Object metadata. The physical content in CAM Element is referred by the attribute "EssenceFileIdentifier" which is a Universal Resource Locator (URL). Actually, the physical content can be referred by the URL. It is worth noting that we do not encapsulate the real media data in the metadata entity but its addressable index, for instance the "torrent" commonly used by current P2P file-sharing systems or a HTTP URL for video sharing in YouTube. This method not only separates metadata processing from content deliver but also makes metadata be light-weight. Moreover, we do not limit the specific way for retrieving media contents used in CHMC. It is open to all existing ways of content delivery.

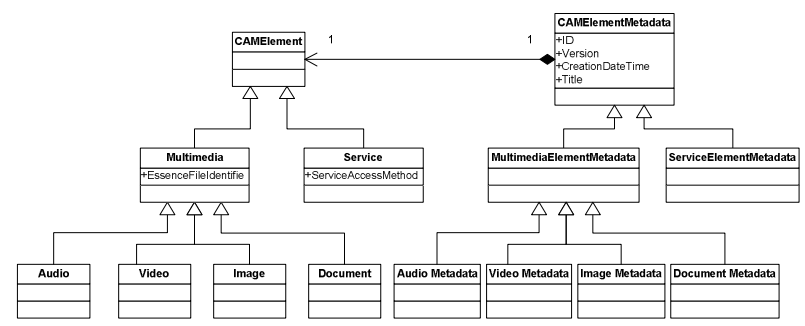

Fig. 1. CAM object metadata.

CAM4Home also supports social metadata that is data generated by collaborative tagging, such as tags, ratings, and comments etc. Examples can be found everywhere on the web, ratings and comments on YouTube, tagging in Digg. The social metadata can help users navigate to relevant contents even quicker because members can use them to provide context and relevant description to the content. However, collaborative tagging differs from traditional metadata in certain important ways $[13,14]$. In order to introduce ambiguous social tagging into structured metadata, the CAM4Home metadata framework defines some prosperities of social metadata that includes social tag, user comment, and user rating.

CAM4Home metadata model is described by RDF Schema (RDFS) [15], and the metadata are instantiated as Resource Description Framework (RDF) [16]. The RDF is used as a general method of modeling information and describing metadata. It can be presented through different syntax formats, where XML is most widely used and supported. The RDF documents are composed of a hierarchy set of atomic RDF statement $(S, P, O)$ which can be regarded as a triple containing subject, predicate and object. The predicate $\mathrm{P}$ describes the relationship between the subject $\mathrm{S}$ and the object $\mathrm{O}$ of the statement.

\section{CHMC SERVICE MODEL AND NETWORK ARCHITECTURE}

Collaborative Home Media Community (CHMC) is a P2Pbased community system for collaboratively sharing, aggregating, and tagging multimedia contents with the use of semantic metadata. The proposed system provides enhanced experiences for users to share multimedia contents encapsulated by semantic metadata on which individual multimedia content can be composited into a semantic bundle for collaboratively delivering. Similar to Web2.0based social tagging service, CHMC also allows users to tag on the shared multimedia contents to facilitate searching, 
organizing, and managing multimedia resources within the community.

Before detailing the technical aspects, we present a scenario to demonstrate the P2P-based CHMC service model. This scenario provides the collaborative multimedia functionalities, such as creation, annotation, searching, retrieval, modification and aggregation. For instance, Jenny went to Paris for sightseeing last week. After return, she created a CAM bundle aggregating a video content and an image content she took in Paris. Then she shared the bundle in CHMC. Jason was going to Paris soon so he did searching on the community and found the entity. After consuming the multimedia contents, Jason rated the entity and tagged each media inside. Mike was shared the entity by his friend Jason. Since he had visited the same places shown in the image, Mike added his comments on it. Alice who was not aware of above behaviors wrote a blog to introduce some good restaurants in Paris. Another user Jeffery thought that this blog may be complementary to the entity. He referred this blog to the CAM entity by adding a link to the blog service. John is a subscriber of IPTV service where there will be a travelling program about Paris a few days later. He aggregated the TV program service in the entity.

The metadata in above scenario can be divided into two types, original metadata and social metadata. The original metadata with the attributes of title, genre, and creation date etc are mainly used to describe multimedia contents or content-based services from the viewpoints of providers or creators. The original metadata entity is generated and verified through the CAM4Home Metadata Processing (MP) web service. Any community member who wants to share multimedia resources has to access the MP Service through a gateway connected with external CAM4Home service platform, where a minimal set of data is required, such as title, description, essence file identifier etc. After that, the entity is shared within CHMC by indexing metadata over the P2P network. Creators or registered users may modify the original metadata by accessing external service platform again, which will update the version number inside of metadata. If so, the original entity will be indexed again within CHMC. The social metadata including tagging, commenting and rating etc., aim to enrich the original metadata by all participators collaboratively. User experiences are enriched and extended on the base of metadata with the collaborations between community members. To prevent malicious acts on the proposed multimedia community, our system does not modify the original metadata directly by inserting social metadata, but distributes the social metadata and associates it with the original metadata. Community members can query on the distributed metadata in order to retrieve multimedia contents.

The proposed system follows a hierarchical P2P architecture where the super-peer model is applied. Fig. 2 illustrates the network architecture of CHMC. A super-peer in the P2P network is a node that operates both as a server to a set of clients and as an equal in a network of super peers [17]. We select super nodes from the home devices with enough computing and storing capacity, as well as highspeed network interface bridging the inner home networks and external Internet. In the home environment, residential gateway [18] is one of the most desirable candidates serving as super nodes. Client nodes are characterized by different properties compared with their counterparts, such as mobility, limited resources, and intermittent connectivity. The array of client nodes envisioned in home includes PC, mobile phones, Personal Digital Assistant (PDA) and Settop Box (STB) etc.

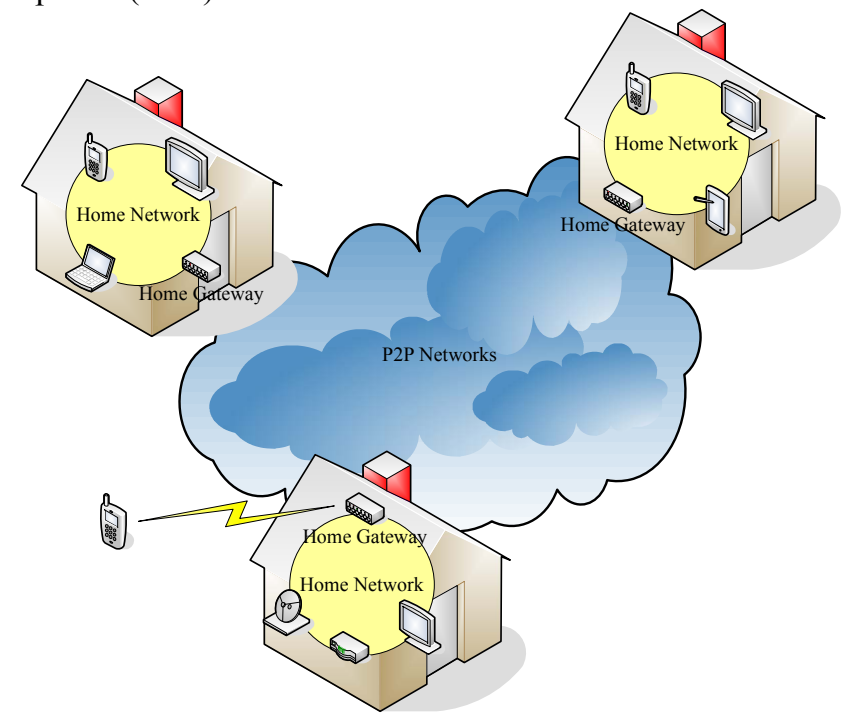

Fig. 2. CHMC network model.

Super nodes are connected with each other maintaining the P2P overlay. On the other hand, client nodes have to connect with and depend on their super nodes in order to get access to the overlay and services provided. Client nodes do not need to run as a P2P node in order to use the service. Instead, they can issue operations to their super nodes that process them on the behalf of client nodes. The advantage of super peer model has three folds. Firstly, peer churn incurred by the dynamic arrival and departure of peers can be reduced to the least, because the home gateway behaving as super node has the uninterrupted connectivity with external Internet. Secondly, user devices connected by the home network are able to involve in CHMC, on behalf by their super nodes. Even, mobile clients outside of home can still access CHMC services through the user's home gateway, as shown by Fig. 2. Thirdly, this way is comparatively lightweight. We can ignore the complexities of deploying a P2P overlay across different types of devices and instead concentrate on developing more sophisticated distributed applications. 


\section{METADATA INDEXING AND RETRIEVING SCHEME}

This section presents a distributed approach to index multimedia contents with the use of CAM4Home semantic metadata. Our approach is based on structured P2P networks and allows semantic queries on multimedia metadata. The structured P2P networks usually referred as Distributed Hash Tables (DHTs) that provide a lookup service similar to a hash table by making available the mapping between a pair (key, value) [19]. The goal of the proposed approach is to provide a fully distributed system that exploits the scalability and efficiency of DHTs in order to index and retrieve multimedia contents through their semantic metadata. It is worth mentioning that this scheme does not aim to build a distributed metadata repository but to index the metadata by RDF triples that are distributed in the DHTbased storage for the purpose of querying or searching.

\subsection{CAM4Home Metadata Indexing}

Metadata indexing means extracting relevant information and associating it with metadata. Aforementioned, CAM4Home metadata, CAM Bundle or CAM Object, are use to present the actual multimedia content. The proposed approach is to provide a fully distributed indexing scheme for CAM Bundle or CAM Object, in order to search, retrieve, and tag multimedia contents. The metadata indexing scheme is composed of two steps. The first step is RDF triple-based iterative indexing algorithm for multimedia addressing and discovery, which provides a mapping for multimedia metadata spread among the distributed peers. It is characterized by a hierarchical scheme in which the metadata are organized through RDF triple. The second step is RDF triple-based storing scheme for metadata querying and searching.

As presented in Section 2, CAM4Home metadata is instantiated as RDF. A RDF document can be decomposed into a hierarchy set of atomic RDF statement. However, there is a discrepancy between metadata representation and storage model in a DHT-based infrastructure [20]. The CAM4Home metadata is serialized as a hierarchical structured $\mathrm{RDF} / \mathrm{XML}$, while the data storage is codified in a flat pair of (key, value). To fill up this gap, we propose a RDF triple-based hierarchical indexing scheme. By the use of hierarchical triples, the CAM metadata can be addressed iteratively and recursively. Take a CAM Object as an example. This object is decomposed into the triple tree $\left\{T_{00}\right.$, $\left.T_{10}, T_{11}, T_{12}, T_{13}, T_{14}, T_{20}, T_{30}\right\}$ as shown by Fig. 3. In order to index CAM4Home metadata, we iteratively put into the DHT overlay a set of (key, value) pair in the form $\left(i d_{m n}, i d_{i j}\right)$. Here, $i d_{i j}$ is generated by hashing the triple $T_{i j}$, while $i d_{m n}$ is done by hashing each descendant of $T_{i j}$. The metadata is identified by $i d_{C A M}$ calculated by the hash function on the metadata itself. The entity $i d_{C A M}$ is associated with $T_{00}$ by $\left(i d_{00}, i d_{C A M}\right)$.
In order to support efficient queries on distributed RDF triples, we exploit an overlay structure to build a distributed index for these triples. Given the generic RDF triple $T_{i j}$ with the form $T_{i j}=\left(S_{i j}, P_{i j}, O_{i j}\right)$, it is deposited into DHT three times by hashing $S_{i j}, P_{i j}$ and $O_{i j}$ respectively, so that the triple $T_{i j}$ can be queried through $S_{i j}, P_{i j}$ or $O_{i j}$. Since the value of attribute "subject" and "predicate" must be a URI which is a string, the hashing function of DHT is directly used to map the values of subject and predicate to the m-bit identifier space. For "object", its type can be URI and literal. In case of sting type, the same hashing function is applied, while the locality preserving hashing in [21] is applied to numeric types. The whole indexing algorithm is presented in Table 1.

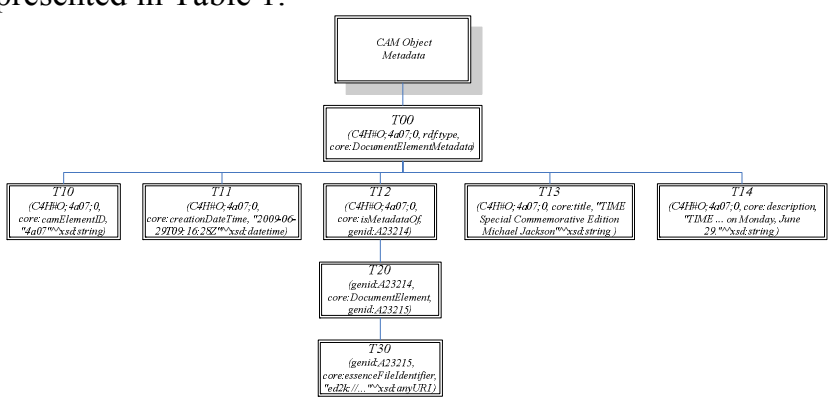

Fig. 3. The RDF triple-based tree Structure of CAM object.

Table 1. CAM4Home metadata indexing algorithm

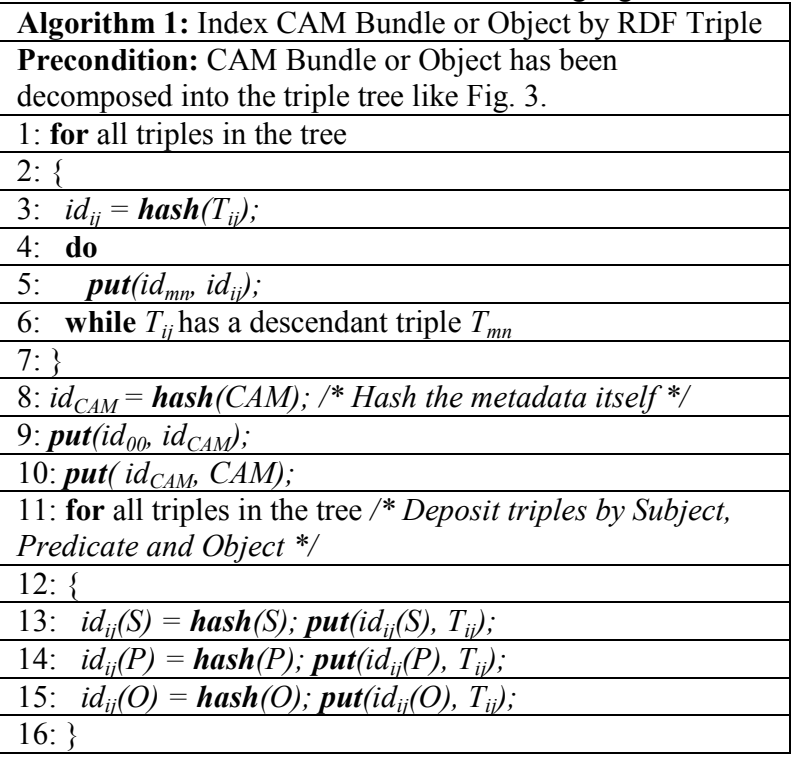

\subsection{Social Metadata Indexing}

In our system, the social metadata are formalized as $\mathrm{RDF} / \mathrm{XML}$ following the CAM4Home RDF Schema. As mentioned in Section 3, the CAM4Home original metadata generated by CAM4Home MP Service is not modified directly when adding social metadata. Instead, the social metadata is distributed over DHTs to index the original metadata. The algorithm presented in Table 2 is adopted to 
insert the social metadata into the RDF triple tree structure of the original CAM Bundle or Object.

Table 2. Social metadata indexing algorithm

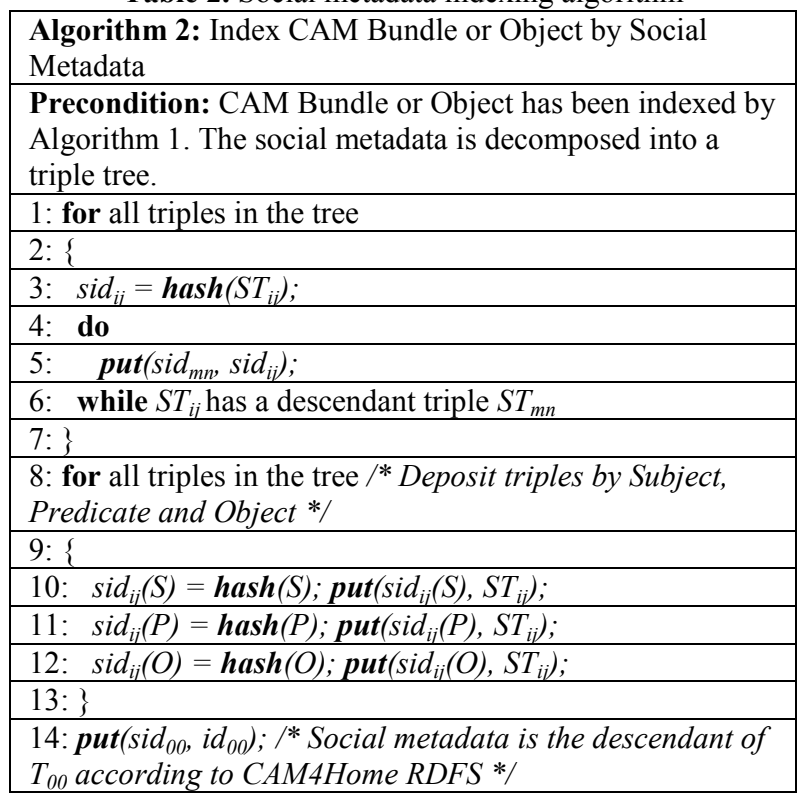

\subsection{Metadata Searching}

In this paper, metadata search is to locate CAM Bundle or Object given one or more their attributes, while metadata query is to extract the required information from metadata. This section focuses on an approach to enable the proposed CHMC system with searching capabilities, which exploits both the triple-based indexing algorithm and knowledgebased RDF query languages. Based on semantic query languages like SPARQL [22] and the triple-based indexing scheme, searching multimedia in the proposed system can be easily achieved as follows. Since RDF triples are distributed on P2P DHT overlay, this paper resolves RDF queries by the way as presented in [23]. As querying results, RDF triples are formed to retrieve CAM Bundle or Object on which they index. For instance, a user wants to search a CAM Object with the social tag "Michael Jackson". Table 3 presents the pseudo-codes of metadata searching algorithm.

Table 3. RDF Triple-based searching algorithm

\begin{tabular}{|l|}
\hline Algorithm 3: CAM Bundle or Object Searching Algorithm \\
\hline Precondition: a) Metadata are indexed by Algorithm 1 and \\
Algorithm 2; b) Search metadata with the social tag value \\
"Michael Jackson" \\
\hline 1: RDF Query \\
\hline 2: SELECT ?SocialTag WHERE \{?SocialTag \\
core:tagValue "Michael Jackson"^^xsd:string\} \\
\hline 3: for all resulting triples \\
\hline 4: \{ \\
\hline 5: key $=$ hash(SocialTag core:tagValue "Michael \\
Jackson"Mxsd:string); \\
\hline 6: Triple = get(key); \\
\hline 7: do \\
\hline 8: $\quad$ index $=$ hash(Triple); \\
\hline 9:
\end{tabular}

\begin{tabular}{|l|}
\hline 10: $\quad$ Triple $=$ get $($ index); \\
\hline 11: $\quad$ while Triple has the parent \\
\hline 12: CAM $=$ get(Triple); \\
\hline 13: \\
\hline
\end{tabular}

\section{PROTOTYPE SYSTEM}

We develop a prototyping system to simulate the proposed CHMC. The system is built on the top of Bamboo DHT [24] that is a proven open source DHT. Bamboo DHT with it public name OpenDHT [25] has been deployed on PlanetLab as a shared DHT service. The super-peer named CAMPeer is developped in a layer-oriented architecture: 1) the underlying DHT overlay is used for message routing and data storing; 2) an application level iterative indexing algorithm for the insertion and retrieval of resources; 3 ) the top CAM4Home Service Layer accessing external CAM4Home service platform for Metadata Processing. To be noted, the implementation exploits a separation model between the DHT overlay and the application layer, which makes CAMPeer independent of any specific DHT. For example, it could be ported to a secure DHT overlay, for the concern of security [26]. Additionally, we simulate a client node with mobile phone powered by J2ME. Client nodes issue requests to its super node by the use of XML Remote Procedure Call (RPC). The super node converts the requests to all CHMC service operations (e.g. indexing, searching and tagging) and processes them on the behalf of mobile clients.

The simulation tests show that the proposed algorithms can be efficiently used to index and retrieve multimedia metadata. We simulate a DHT overlay with 50 super nodes. Table 4 presents the time performance. To a normal CAM Object constituted by 13 triples, there are 57 times of "Put" indexing the object. It takes $104562 \mathrm{~ms}$ in average to index the metadata object, among which $98 \%$ of the time is in fact spent to put triples into the Bamboo DHT. The latencies for querying and searching are much shorter than that of indexing. It can be explained that they do much fewer "Get", for instance 2 times for (? P O) querying. We further observe the average latency for "Put" is $1888 \mathrm{~ms}$, while $893 \mathrm{~ms}$ for "Get", which are consistent with results of the large-scale test in [27].

Table 4. Time performance

\begin{tabular}{|c|c|c|}
\hline $\begin{array}{c}\text { Metadata } \\
\text { Indexing }\end{array}$ & $\begin{array}{c}\text { (? P O) } \\
\text { Query }\end{array}$ & $\begin{array}{c}\text { Search by } \\
\text { Triple }\end{array}$ \\
\hline $104562 \mathrm{~ms}$ & $1760 \mathrm{~ms}$ & $1786 \mathrm{~ms}$ \\
\hline
\end{tabular}

\section{CONCLUSIONS}

Collaborative use of multimedia enables people to experience more and share more with other people. In this paper, we have presented the novel concept of P2P-based collaborative home multimedia community, with the vision of sharing, aggregating and searching media contents on the base of rich semantic metadata. Making use of the structured 
P2P overlay as the network infrastructure for multimedia sharing, the proposed system is characterized by scalability, collaboration, aggregation, social tagging, and semantic metadata. We propose a distributed approach to index multimedia contents with the use of CAM4Home semantic metadata. Multimedia contents to be shared are encapsulated as semantic metadata which are indexed by RDF triples for query and search. The collaborative tagging is distributed over the P2P networks and used to index the original metadata. The simulation test shows that the proposed algorithms can be efficiently used to index and retrieve multimedia content.

\section{REFERENCES}

[1] Y. Rasheed, "High-Quality Media Distribution in the Digital Home," Intel Technology Journal, vol. 06, pp. 17, Nov. 2002.

[2] UPnP, http://www.upnp.org.

[3] DLNA, http://dlna.org.

[4] D.H. Kim, V.C. Joseph, K. Lee, and E.H. Jo, "A Hybrid Architectural Framework for Digital Home Multimedia Multimodal Collaboration Services," Advances in Multimedia Information Processing, 2004.

[5] J.T. Kim, Y.J. Oh, H.K. Lee, E.H. Paik, K.R. Park, "Implementation of the DLNA Proxy System for Sharing Home Media Contents", Proceedings of International Conference on Consumer Electronics, 2007.

[6] J. Sung, D. Kim, H. Song, J. Kim, S.Y. Lim, J.S. Choi, "UPnP based Intelligent Multimedia Service Architecture for Digital Home Network", Software Technologies for Future Embedded and Ubiquitous Systems, 2006.

[7] J. Hjelm, T. Oda, A. Fasbender, S. Murakami, A. Damola, "Bringing IMS Services to the DLNA Connected Home", The $6^{\text {th }}$ International Conference on Pervasive Computing, May 2008.

[8] E. Turcan, L. Stromback, J. Morris, "Share it! by Bringing P2P into the TV-Domain", Third International Conference on Peer-toPeer Computing, 2003.

[9] J. Walker, O. J. Morris, B. Marusic, "Share it! - The Architecture of a Right-managed Network of Peer-to-Peer Set-topboxes", EUROCON 2003, Ljubljana, Slovenia.

[10] P2P-FUSION, http://p2p-fusion.org/.

[11] P2P-Next, http://www.p2p-next.org/.

[12] CAM4Home, http://www.cam4home-itea.org/.

[13] S. A. Golder, and B. A. Huberman, "Usage Patterns of Collaborative Tagging Systems", Journal of Information Science, vol. 32, pp. 198-208, 2006.

[14] John C. Paolillo, and S. Penumarthy, "The Social Structure of Tagging Internet Video on del.icio.us", Proceedings of the $40^{\text {th }}$ Hawaii International Conference on System Sciences, 2007.

[15] Resource Description Framework (RDF) Schema, http://www.w3.org/TR/rdf-schema/.

[16] Resource Description Framework (RDF), http://www.w3.org/RDF/

[17] B. Yang, H. Garcia-Molina, "Designing a Super-Peer Network", the $19^{\text {th }}$ International Conference on Data Engineering, 2003.

[18] Home Gateway Initiative, http://www.homegateway.org.

[19] H. Balakrishnan, M. F. Kaashoek, D. Karger, R. Morris, I. Stoica, "Looking up Data in P2P Systems", v. 46, no. 2, pp. 43-48, Communication of the ACM, Feb. 2003.
[20] W. Allasia, F. Gallo, M. Milanesio, and R. Schifanella, "Indexing and Retrieval of Multimedia Metadata on a Secure DHT", Informatica - Special Issue on Multimedia Information System Security, Vol. 33, No. 1, pp. 850-100, March 2009.

[21] M. Cai, and M. Frank, "MAAN: A Multi-attribute Addressable Network for Grid Information Services", Proceeding of $4^{\text {th }}$ International Workshop on Peer-to-Per Systems, Feb. 2003.

[22] SPARQL Query Language for RDF, http://www.w3.org/TR/rdf-sparql-query/.

[23] M. Cai, M. Frank, "RDFPeers: A Scalable Distributed RDF Repository based on A Structured Peer-to-Peer Network", Proceedings of the $13^{\text {th }}$ International Conference on World Wide Web, pp. 650-657, 2004

[24] Bamboo DHT, http://bamboo-dht.org/index.html.

[25] S. Rhea, B. Godfrey, B. Karp, J. Kubiatowicz, S. Ratnasamy, S. Shenker, I. Stoica, and H. Yu, "OpenDHT: A Public DHT Service and Its Uses", Proceedings of ACM SIGCOMM 2005, August 2005.

[26] G. Urdaneta, G. Pierre, M. V. Steen, "A Survey of DHT Security Techniques”, ACM Computing Surveys, 2009.

[27] B. Meyer, M. Portmann, "Practical Performance Evaluation of Peer-to-peer Internet Telephony using SIP", IEEE $8^{\text {th }}$ International Conference on Computer and Information Technology Workshops, July 2008. 